(2) Open Access Full Text Article

REVIEW

\title{
Measuring disease activity in Crohn's disease: what is currently available to the clinician
}

This article was published in the following Dove Press journal:

Clinical and Experimental Gastroenterology

20 May 2014

Number of times this article has been viewed

\section{Renata D'Incà \\ Roberta Caccaro \\ Department of Surgical, Oncological and Gastroenterological Sciences, Gastroenterology Section, University of Padua, Padua, Italy}

\begin{abstract}
Crohn's disease (CD) is a chronic inflammatory bowel disease characterized by a relapsing-remitting clinical behavior and dominated by intestinal inflammation. Being a chronic disorder that with time develops into a disabling disease, it is important to monitor the severity of inflammation to assess the efficacy of medication, rule out complications, and prevent progression. This is particularly true now that the goals of treatment are mucosal healing and deep remission. Endoscopy has always been the gold standard for assessing mucosal activity in $\mathrm{CD}$, but its use is limited by its invasiveness and its inability to examine the small intestine, proximal to the terminal ileum. Enteroscopy and the less invasive small bowel capsule endoscopy enable the small bowel to be thoroughly explored and scores are emerging for classifying small bowel disease activity. Cross-sectional imaging techniques (ultrasound, magnetic resonance, computed tomography) are emerging as valid tools for monitoring CD patients, assessing inflammatory activity in the mucosa and the transmucosal extent of the disease, and for excluding extra-intestinal complications. Neither endoscopy nor imaging are suitable for assessing patients frequently, however. Noninvasive markers such as C-reactive protein, and fecal biomarkers such as calprotectin and lactoferrin, are therefore useful to confirm the inflammatory burden of the disease and to identify patients requiring further investigations.
\end{abstract}

Keywords: activity, biomarkers, Crohn, endoscopy, imaging, monitoring

\section{Introduction}

Crohn's disease (CD) is a chronic inflammatory bowel disease characterized by a relapsing-remitting clinical behavior. The main feature of CD is intestinal inflammation. Its site, extent, and severity influence the phenotype and natural history of the disease (together with factors such as age at onset, smoking habits, perianal disease, and the need for steroids at diagnosis). ${ }^{1,2}$ It is not unusual to find intestinal inflammation (even without any gastrointestinal symptoms) leading to progressive bowel damage, increasing disability, and an impaired quality of life. ${ }^{3}$ In recent years, the goals of treatment have gradually moved beyond clinical remission towards a new (and as yet poorly defined) concept termed "sustained deep remission", which means a condition of clinical, biological, endoscopic, and possibly, radiological remission. ${ }^{4,5}$ Measuring clinical activity is consequently still important, but no longer enough, and the available symptom-based scoring systems (the Crohn's Disease Activity Index [CDAI], and the simpler Harvey Bradshaw Index) suffer from subjective interpretation. ${ }^{6-8}$ The high rates of response to placebo (up to $33 \%$ ) reported in randomized controlled trials using the CDAI to measure response to treatment justify the need for more objective methods for assessing the presence and severity of intestinal inflammation. ${ }^{9}$ The ultimate goal
Correspondence: Renata D'Incà Department of Surgical, Oncological and Gastroenterological Sciences, Gastroenterology Section,

University of Padua, Via Giustiniani 2,

35 I 28 Padova, Italy

Tel +39498212890

Fax +39498760820

Email dinca@unipd.it 
of monitoring in $\mathrm{CD}$ patients is to modify the natural history of the disease, taking a "treat-to-target" approach. ${ }^{10}$

This review provides a brief, practical overview of the tools currently available to clinicians for objectively measuring disease activity in $\mathrm{CD}$ patients. In particular, we focus on the ability of endoscopy, cross-sectional imaging techniques, and biomarkers to detect intestinal inflammation.

\section{Endoscopy}

Ileocolonoscopy is considered the gold standard for the diagnosis and assessment of disease activity and extent in $\mathrm{CD}$ patients. It has recently increased in importance with the use of biologics, and the growing awareness that mucosal healing may be the optimal treatment target with a view to changing the natural history of the disease. ${ }^{11-13}$

It is well known that clinical symptoms of CD seem to correlate little with the severity of endoscopic lesions, ${ }^{14}$ while it has been demonstrated that the severity and extent of mucosal lesions influence the course of the disease. Allez et al reported that finding severe endoscopic lesions (deep ulcerations covering more than $10 \%$ of the mucosal area of at least one segment of the colon) at colonoscopy in 102 patients with $\mathrm{CD}$ was a risk factor for penetrating complications and colectomy, with a probability of colectomy at 1,3 , and 8 years of $31 \%, 42 \%$, and $62 \%$, respectively. ${ }^{15}$ In another study, a stricturing phenotype predicted a poor response to medical treatment and a greater need for surgery. ${ }^{16}$ Complete mucosal healing in newly diagnosed $\mathrm{CD}$ patients after 2 years of medical treatment emerged as the only factor capable of predicting sustained, steroid-free remission for up to 4 years. ${ }^{12}$ Similarly, in a large Norwegian population-based prospective cohort study, mucosal healing 1 year after the diagnosis of $\mathrm{CD}$ (in the prebiologic era) predicted a more limited subsequent disease activity and need for active treatment. ${ }^{17}$

Endoscopic remission should therefore be considered an early target of medical treatment. Af Bjorkesten et al showed that endoscopic remission after 3 months of antitumor necrosis factor alpha treatment strongly predicts endoscopic remission at 1 year. ${ }^{18}$ The benefit of reaching mucosal healing is also confirmed by the effects of longterm maintenance infliximab treatment, with an improved long-term disease outcome, less major abdominal surgery, fewer hospitalizations, and fewer relapses. ${ }^{11,19}$ Much the same can be said of adalimumab, judging from the results of the EXTEND trial. ${ }^{13}$

Endoscopy is thus a crucial step in assessing CD activity, but it has some limitations: it is invasive, poorly accepted by patients, and it can only examine the mucosa, not the deeper layers of the intestinal wall, and it misses any extra-intestinal complications of the disease. ${ }^{20}$ There is also some debate on the definition of mucosal healing, and how much a partial improvement in endoscopic disease activity in response to treatment suffices to improve clinical outcome remains to be seen. ${ }^{21,22}$ In some studies, even a partial healing of the mucosa was enough to improve the course of the disease. ${ }^{11,18}$ The most appropriate schedule for the endoscopic follow-up of $\mathrm{CD}$ activity also remains unclear. For the time being, it should be planned on a case by case basis, when the findings of an endoscopy might influence the management of a given patient's disease.

Endoscopic scoring systems have been developed to classify disease activity and they have been validated to enable a consistent and reproducible assessment of CD activity, minimizing interobserver variability. There are currently three endoscopic scoring systems for CD: the Crohn's Disease Endoscopic Index of Severity (CDEIS), ${ }^{23}$ the Simple Endoscopic Score for Crohn's Disease (SES-CD), ${ }^{24}$ and the Rutgeerts endoscopic grading scale..$^{25}$

The CDEIS scores CD activity (from 0 to 44 ) in five bowel segments (terminal ileum, right colon, transverse, left colon and sigmoid, and rectum), considering specific mucosal lesions such as ulcers and stenosis, and extent of disease. ${ }^{21-23}$ The CDEIS is complicated to use, requiring training and experience in estimating the extent of ulcerated or diseased mucosal surfaces, and expertise in distinguishing deep from superficial ulcerations. It is also time consuming. It has consequently not become routine in clinical practice and is used mainly in clinical trials.

To simplify the CDEIS and to make it easier to use in every day clinical practice, Daperno et al developed the SESCD. ${ }^{24}$ This system includes four variables, each considered in five segments of the bowel: ulcer size $(0.1-0.5 \mathrm{~cm}$; $0.5-2 \mathrm{~cm} ;>2 \mathrm{~cm})$, the extent of ulcerated surface $(<10 \%$; $10 \%-30 \% ;>30 \%)$, the extent of affected surface $(<50 \%$; $50 \%-75 \% ;>75 \%$ ), and stenosis (single or multiple, and partially or totally occluded). Scores range from 0 to $60 .{ }^{21,22,24}$ The SES-CD significantly correlates with the CDEIS (Spearman's $r=0.938, P<0.0001$ ), and this correlation is also maintained when the disease is graded from inactive to severe, and when changes in activity are compared between baseline and follow-up endoscopies. ${ }^{26}$

Endoscopic scores are useful objective tools for assessing the degree of CD activity, and should be used to monitor disease evolution and orient treatment decisions. The degree of mucosal improvement (endoscopic response) needed to alter midterm outcome is not known, however. Ferrante et al 
recently performed a post hoc analysis on data from the SONIC trial addressing this specific issue. ${ }^{27}$ In 172 patients they studied the minimum clinically important improvement in endoscopic disease activity at week 26 that reliably predicted corticosteroid-free clinical remission at week 50 , and could therefore be proposed as an appropriate cutoff for endoscopic response. A reduction of at least $50 \%$ in the SESCD or CDEIS score after 26 weeks of treatment identified the patients most likely to be in corticosteroid-free clinical remission at week 50 .

The situation of $\mathrm{CD}$ patients after surgery deserves a separate discussion. Given the finding that almost $80 \%$ of patients have an endoscopically detectable recurrence within 1 year after surgery (even without clinical symptoms), ${ }^{28,29}$ Rutgeerts et al developed a score for grading lesions in the neo-terminal ileum and anastomosis. ${ }^{25}$ This score ranges from 0 to 4: 0 , no lesions; $1,<5$ aphthous lesions; $2,>5$ more severe aphthous lesions; 3 , diffuse inflammation with diffuse ulcers; and 4, nodules and/or narrowing. This score is considered the gold standard for establishing the prognosis in cases of postoperative recurrence: scores 3 and 4 are validated cutoffs for predicting clinical relapse, and should prompt medical treatment to prevent disease evolution and clinical relapse.

The goal of optimal treatment should be to stop the inflammatory process completely. Mucosal healing may not suffice and histological healing should be considered the final target, but there is currently no accepted definition of histological healing, or standardized histological scoring system. The presence of neutrophils in the lamina propria and epithelium, epithelial cell damage, and an increase in lymphocytes and plasma cells are considered features of disease activity, and their persistence may be associated with relapse. ${ }^{30}$ It is important to bear in mind that histological healing does not necessarily coincide with mucosal healing: D'Haens et al showed that CDEIS and inflammatory infiltrate improved in patients treated with a single infusion of infliximab, but architectural glandular changes persisted in most patients. ${ }^{31}$ Smith et al showed that CD patients on prolonged treatment with naltrexone further improved in terms of their endoscopic inflammation scores beyond the 12 week follow-up, but this was not associated with any further improvement in their histological scores. ${ }^{32}$

One of the limits of conventional endoscopy in $\mathrm{CD}$ is that the small bowel remains inaccessible. Small bowel endoscopy is defined as any endoluminal examination of the small bowel, including capsule endoscopy, push enteroscopy and balloonassisted endoscopy. ${ }^{33}$
Small bowel capsule endoscopy (SBCE) enables the whole of the small bowel to be explored using a wireless capsule, which is swallowed and propelled through the gastrointestinal tract by gut motility. According to the recently published international OMED-ECCO consensus, there are no specific SBCE diagnostic criteria for CD: the method has a high diagnostic yield in terms of identifying small bowel mucosal lesions (even better than magnetic resonance imaging [MRI] or computed tomography [CT], judging from a few preliminary findings). ${ }^{34,35}$ Such endoscopic lesions may not be specific for $\mathrm{CD}$, however, and having no opportunity to perform a histological examination prevents any conclusive differential diagnosis. In patients with an established diagnosis of CD, SBCE should be reserved for cases with unexplained symptoms, when conventional ileocolonoscopy and imaging techniques prove inconclusive, and when identifying less obvious superficial lesions may have an impact on a patient's clinical management. ${ }^{33}$

Recent evidence has pointed to a role for SBCE in postoperative patient management to detect lesions in the small bowel overlooked by ileocolonoscopy after an ileocolic resection. ${ }^{36}$ Ileocolonoscopy nonetheless remains the gold standard for assessing postoperative recurrences. The use of SBCE to monitor response to therapy is still controversial. As already established for conventional endoscopy, so too for SBCE, clinical activity does not correlate with mucosal healing. Efthymiou et al found clinical response apparently unrelated to mucosal healing as detected by capsule endoscopy, since only one of three variables (the number of large ulcers) improved significantly with treatment. ${ }^{37}$ Similarly, Yang et al found that clinical and biological improvements after medical treatment were not consistently associated with any amelioration in the mucosal lesions seen at SBCE. ${ }^{38}$

Disease activity indices have been proposed for use with SBCE with a view to making the assessment and monitoring of mucosal healing more objective. The Capsule Endoscopy CD Activity Index ([CECDAI] or Niv score) was validated in a multicenter prospective study on 62 patients with isolated small bowel CD. ${ }^{39}$ The three main parameters considered were inflammation, extent of disease, and presence of strictures. Then there is the Lewis score, which assesses villous edema, ulcer and stenosis, classifying CD activity from mild to severe. ${ }^{40}$ When the performance of these two SBCE inflammation scoring systems (CECDAI and Lewis score) was tested by correlating them with patients' fecal calprotectin levels, the Lewis score correlated better with fecal calprotectin levels, providing they were below $100 \mu \mathrm{g} / \mathrm{g} .{ }^{41}$ These SBCE scoring systems have been 
developed only recently, and their usefulness in clinical trials and clinical practice remains to be seen. ${ }^{21}$

Balloon-assisted enteroscopy (using double or single balloons) has largely replaced push enteroscopy because it enables the small bowel to be intubated completely via oral and anal routes and, unlike SBCE, it allows for tissue sampling and therapeutic procedures. The disadvantages of this technique lie in its complexity and the need for patient sedation and for specifically trained and experienced endoscopists to perform it. The method is not widely used. ${ }^{33} \mathrm{~A}$ summary of advantages and disadvantages of endoscopy for the monitoring of CD is reported in Table 1.

\section{Cross-sectional imaging techniques}

Although endoscopy remains the gold standard for assessing CD activity, it suffers from several limitations. Colonoscopy only explores the colon and a few centimeters of the terminal ileum. Gastroscopy is helpful in only a minority of patients. Double balloon enteroscopy is not widely available and rather invasive. As for SBCE, no biopsies can be obtained, it is not advisable in cases of stenosis, and it may provide suboptimal information on the small bowel if patient preparation is inadequate. In addition, endoscopic procedures can only examine the superficial layer of the intestinal mucosa, missing

Table I Advantages and disadvantages of endoscopic techniques for monitoring Crohn's disease activity

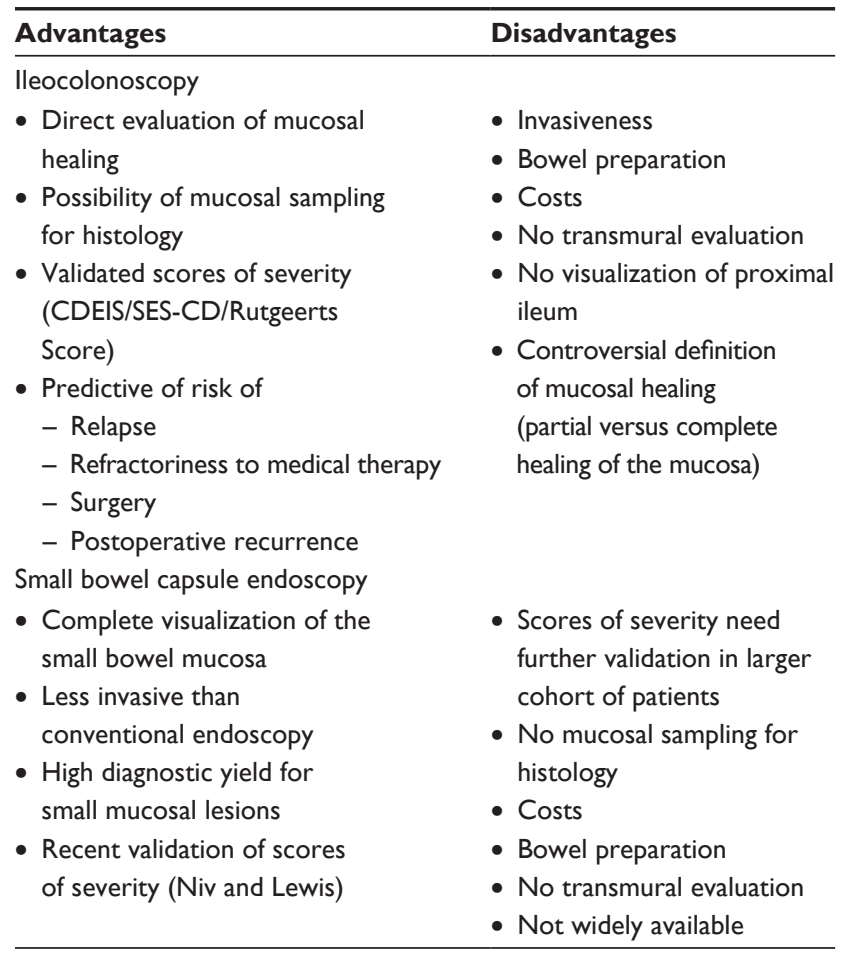

Abbreviations: CDEIS, Crohn's Disease Endoscopic Index of Severity; SES-CD, Simple Endoscopic Score for Crohn's Disease. transmural and extra-intestinal manifestations of the disease. Cross-sectional imaging techniques are therefore gaining in importance in a complementary role, especially to measure the cumulative intestinal damage and the interplay between inflammation and fibrosis. A summary of advantages and disadvantages of cross-sectional imaging techniques for the monitoring of CD is reported in Table 2.

\section{Ultrasound (US)}

US is a noninvasive, radiation-free imaging technique that is particularly useful for examining the terminal ileum and colon, whereas the proximal ileum, jejunum, transverse colon, and rectum may be difficult to assess. ${ }^{42}$ Visualization of the intestinal wall may be limited by gas-filled bowel and obesity, and the diagnostic accuracy of US is also influenced by the sonologist's experience. ${ }^{42,43}$

The main features detectable on US bowel studies are bowel wall thickness, loss of stratification, strictures, wall stiffness with a reduced or no peristalsis in the small bowel, and loss of haustra coli in the colon. ${ }^{44,45}$ Other US findings correlating with $\mathrm{CD}$ include poor compressibility

Table 2 Advantages and disadvantages of cross-sectional imaging techniques for monitoring Crohn's disease activity

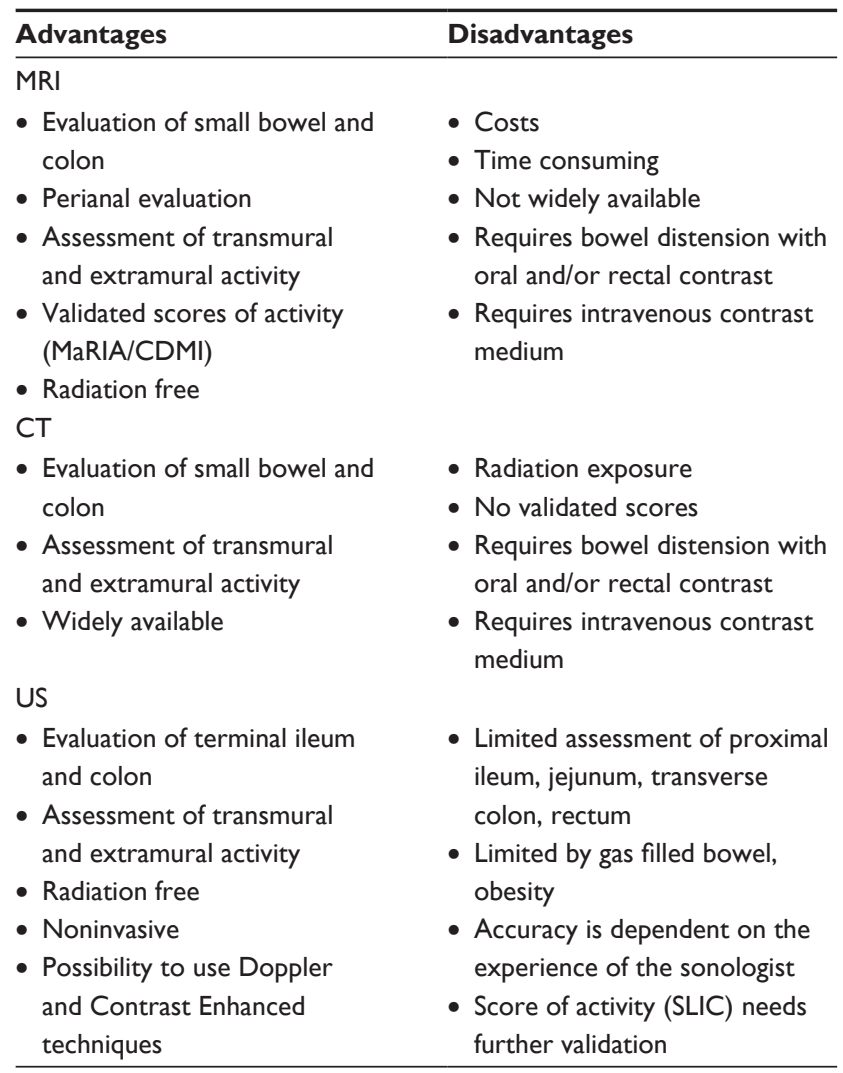

Abbreviations: CDMI, Crohn's Disease MRI Index; CT, computed tomography; MaRIA, Magnetic Resonance Index of Activity; MRI, magnetic resonance imaging; SLIC, sonographic lesion index for CD; US, ultrasound. 
of thickened bowel walls, narrowing of the lumen, conglomeration of loops, and extramural lesions such as fistulae or abscesses. $^{43}$

Nonabsorbable oral solutions can be administered to increase the sensitivity of US in detecting segments with active disease, and using a luminal contrast in the colon can improve its accuracy in assessing colonic CD lesions. ${ }^{43,46,47}$ In the systematic review conducted by Panes et al, the reported overall sensitivity and specificity of US in assessing CD activity were $85 \%$ and $91 \%$, respectively. ${ }^{43}$ Doppler US and contrast-enhanced US may feasibly help emphasize the vascularization pattern and thus increase the method's diagnostic accuracy in identifying CD activity, but they have demonstrated a similar sensitivity and specificity to conventional US. ${ }^{48}$ On the other hand, contrast-enhanced US was able to classify the severity identified at endoscopy significantly better than Doppler US. ${ }^{49,50}$ Calabrese et al developed a quantitative index for assessing CD-related small bowel damage as detected by small intestine contrast ultrasonography, called the sonographic lesion index for CD (SLIC). ${ }^{51}$ The SLIC considers both the extent and the severity of small bowel damage (bowel wall thickness, lumen diameter, length of lesions, and number of sites involved), as well as fistulas, mesenteric adipose tissue changes, abscesses, and lymph nodes. It has the potential for scoring the progression of small bowel disease by means of serial assessments, and of quantifying any changes after treatments. It can therefore be a useful, noninvasive tool for gastroenterologists with sufficient expertise. This score needs to be validated on a larger cohort of patients, however, before it can enter clinical practice.

\section{CT}

CT is a valid tool for examining luminal and extraluminal structures in CD patients, and it has the advantage of being widely available and standardized in its use. ${ }^{42,43}$ Its major drawback lies in the related radiation exposure, especially in patients being assessed repeatedly.

CT enterography requires an adequate luminal distension obtained by means of oral contrast agents (such as polyethylene glycol solutions), and the intravenous administration of contrast medium is essential to assess bowel wall enhancement and mesenteric vessels. ${ }^{52}$ Typical CT findings of active $\mathrm{CD}$ are mural hyperenhancement, bowel wall thickening ( $>3 \mathrm{~mm}$ ), mural stratification due to intramural edema, engorged vasa recta (comb sign), and increased attenuation of mesenteric fat. The CT features that may be helpful in differentiating active inflammatory disease from a fibrostenosing evolution of CD include strictures without mural hyperenhancement, submucosal fat deposition, and absence of mural stratification due to transmural fibrosis. ${ }^{52}$ Drawing this distinction may be difficult, however, because active inflammation and fibrosis often coexist in the same patient, and even in the same bowel segment.

CT enterography is highly sensitive and specific in detecting intestinal lesions, especially in the ileum, ${ }^{53}$ with an overall sensitivity and specificity of around $81 \%$ and $88 \%$, respectively. It is less effective in detecting colonic lesions, and proper administration of the contrast is very important. An amelioration in the typical CT findings of active CD, or even their disappearance, after a course of treatment correlates with the achievement of a clinical remission, as demonstrated in a retrospective study on $50 \mathrm{CD}$ patients. ${ }^{54}$

When CT and MRI were compared in terms of their diagnostic accuracy, neither proved significantly superior to the other. They both provide complementary information to ileocolonoscopy, especially when exploration of the ileum is not feasible..$^{53,55-57}$

In the management of CD patients, it has been suggested that CT is more appropriate in acute cases and older patients, partly to contain radiation exposure, and partly because it requires fewer breath holds (than MRI), improving patient compliance. The use of the latest generation of multidetector CT scanning and low dose protocols has recently significantly reduced the radiation dose involved. Although these protocols produce lower quality images, this does not affect the technique's diagnostic value. ${ }^{58,59}$

Cipriano et al recently developed a model to compare the life time costs, benefits, and cost effectiveness of using MRI rather than CT for routine disease monitoring in young CD patients. ${ }^{60}$ They demonstrated that although MRI is more costly than $\mathrm{CT}$, it remains a cost effective alternative to $\mathrm{CT}$ for younger patients with $\mathrm{CD}$ who are likely to require multiple imaging procedures over the course of their disease. This result appeared to be related to a reduction in the cancer risk related to radiation exposure. ${ }^{60}$

\section{MRI}

MRI is gaining a major role for its diagnostic accuracy in the management of CD patients. Using a radiation free method is particularly important in young patients needing numerous examinations during their life time. For assessing CD activity, there are specific MRI features that can be measured with standard protocols, acquiring fast, unenhanced T2-weighted and gadolinium-enhanced T1-weighted sequences. ${ }^{61,62}$ These acquisition protocols enable images to be obtained 
within a single breath hold, with limited motion and peristaltic artifacts, while an intravenous injection of contrast medium reveals the enhancement pattern of the bowel wall and mesenteric vessels. Adequate bowel distension is essential to obtain good quality images and to avoid missing less obvious signs, such as ulcerations. This distension can be achieved by administering enteric contrast agents either orally (magnetic resonance [MR] enterography) or through a nasojejunal tube (MR enteroclysis). ${ }^{63,64}$ Enterocolonography also demands colon distension with a water enema, and provides information on the whole gastrointestinal tract. Special MRI sequences and techniques can be used to further improve the quantitative and qualitative diagnostic capability of MRI in ascertaining CD activity and severity, ${ }^{65,66}$ including diffusion-weighted imaging, motility imaging, and magnetization transfer imaging.

The MRI features measured to assess CD activity mainly concern mural and mucosal characteristics, including the presence of ulcerations, wall thickness, mural T2 signal intensity, and T1 contrast enhancement. ${ }^{65}$ There are also extramural features of inflammation, such as a comb sign, lymph node enlargement, and fat wrapping. ${ }^{65}$

Combining certain MRI features into a scoring system may be the best way to improve the accuracy of MRI in measuring CD activity. ${ }^{67}$ Rimola et al proposed and validated a simplified Magnetic Resonance Index of Activity (MaRIA) score that quantifies CD-related inflammatory activity in each ileocolonic segment $(1.56 \times$ wall thickness in millimeters $+0.02 \times$ relative contrast enhancement $+5 \times$ edema $+10 \times$ ulceration). ${ }^{68,69}$ Wall thickness in millimeters $(P=0.007)$, relative contrast enhancement $(P=0.01)$, mural edema $(P=0.02)$, and ulceration at MRI $(P=0.003)$ emerged as independent predictors of segmental CDEIS $(r=0.82, P<0.001) .{ }^{68}$ It should be noted, however, that the MRI in the two above cited studies, ${ }^{68,69}$ was performed with a 3.0 Tesla MR unit, and that the protocol required the use of both oral contrast and colon enema to distend the small bowel and colon, respectively. It is hard to say how reproducible the MaRIA score may be in patients undergoing only standard MRI without dedicated colon preparation. ${ }^{65}$

Another MRI score is the Crohn's Disease MRI Index (CDMI), which considers mural thickness, mural T2 signal, perimural T2 signal, and mural T1 enhancement. These features (scored from 0 to 3 ) have proved to be significantly correlated to a reference standard of transmural pathological activity grading in surgical resection specimens. When the score was validated vis-à-vis endoscopic biopsy in a cohort of patients, its sensitivity was $81 \%$ and its specificity $70 \%$ for histopathological activity. ${ }^{70}$

For an imaging technique to be used routinely in the management of CD patients, reproducibility and a low interobserver variability are essential requirements. Tielbeek et al recently assessed interobserver variability across four readers for scoring MRI features and scoring systems (MaRIA and CDMI) in patients with CD, using CDEIS as the reference standard. ${ }^{71}$ Wall thickness in millimeters, the presence of edema, enhancement pattern, and length of sites of disease in each segment showed good reproducibility, but for extramural MRI features (perimural T2 signal, comb sign, and lymph nodes) only fair reproducibility was observed (the absence of severe patients may have influenced the results). When individual features were combined with the scoring systems, the interobserver variability was good; the correlation with the CDEIS only moderate. The MRI protocol was different from the one used by Rimola, however. ${ }^{68,69}$

The way forward is to use MRI enterography to monitor treatment response after a flare of $\mathrm{CD}$, aiming to achieve transmural healing as well as mucosal healing, which has been correlated with a better CD course, fewer hospital admissions, less surgery, and higher steroid-free remission rates. ${ }^{4,11,12}$ This new treatment goal is particularly important because mural inflammation may persist even in cases of mucosal healing.

The study by Sempere et al found that a significant decrease in wall thickness and contrast enhancement of the affected bowel wall coincided with a change from the active disease phase to clinical remission. ${ }^{72}$ In particular, once clinical remission had been achieved, there was no significant difference in the mean contrast enhancement between $\mathrm{CD}$ patients and controls, but intestinal segments remained thicker in $\mathrm{CD}$ patients in remission than in healthy controls. Ordàs et al used the MaRIA score to monitor response to therapy in 27 patients after 12 weeks of corticosteroid or adalimumab therapy (CDEIS was used as the reference standard). ${ }^{73}$ A MaRIA score below 40 predicted endoscopic remission with a sensitivity of $82 \%$ and a specificity of $85 \%$. Van Assche et al followed up 15 patients on infliximab therapy by performing MRI enteroclysis at weeks 2 and $26 .{ }^{74}$ The inflammatory components of the MRI index had improved at week 2, correlating with the CDAI, but complete normalization of MRI findings was rare.

Assessing MRI features could also help to predict future response to treatment. Lawrance et al suggested classifying CD patients based on the following MRI findings: 1) fibrosis 
(bowel wall thickening without contrast enhancement); 2) mild segmental hyperenhancement and mild wall thickening; 3) mild segmental hyperenhancement and marked wall thickening; and 4) marked segmental transmural hyperenhancement. ${ }^{75}$ They demonstrated that female patients and those in the mild segmental hyperenhancement category were more likely to respond to medical therapy (based on the Harvey Bradshaw Index), while patients with luminal narrowing and hold up were less likely to do so $(P<0.05)$. Inflammation and fibrosis are closely and positively related, however, sometimes making it difficult to distinguish between them and to exclude one or the other, as they often coexist. ${ }^{76,77}$

The panoramic view they provide of the whole abdomen makes cross-sectional imaging techniques important diagnostic tools for assessing complications in CD. MRI is considered slightly more sensitive and specific in diagnosing intestinal stenosis than CT or US. ${ }^{20,42,43}$ Accuracy in diagnosing intra-abdominal fistulas and abscesses is comparable for MRI and CT, both achieving high levels of sensitivity and specificity.

Pelvic CD is one of the clinical settings in which the superiority of MRI is unquestioned. It is more effective than CT in detecting fistulizing tracts and elucidating their relationship with the anal sphincter and adjacent structures in the perianal soft tissues, especially in cases of complex fistulae. ${ }^{66}$ Proper anatomical assessment of perianal disease is particularly important to orient the patient toward the best therapeutic approach, which is medical rather than surgical.

Van Assche et al developed an MRI-based score of perianal CD severity, based on the local extent of fistulae (number of tracks, location in relation to the anal sphincter, extension) and active inflammation (hyperintensity, presence of collections and rectal wall involvement), to assess the anatomical evolution of perianal fistulae. ${ }^{78}$ This score was first applied to 18 patients before and after treatment with infliximab, and it proved reliable in assessing fistula tracks, with good interobserver agreement $(P<0.001)$. The study showed that despite closure of draining external orifices after infliximab therapy, fistula tracks persisted with varying degrees of residual inflammation, causing recurrent fistulae and pelvic abscesses. Long-term monitoring of infliximab therapy for perianal fistulizing CD revealed that MRI can only be considered reliable for monitoring the first year of treatment; in the longer term, clinical assessment is equally reliable or even superior to MRI for the purpose of assessing the outcome of therapy, and it would save health care resources (given the cost of repeated MRI). ${ }^{79}$

\section{Biomarkers}

Endoscopy and imaging techniques are the most objective methods for assessing CD activity, but they have their limitations and risks. Endoscopy is invasive and requires bowel cleansing, a procedure that is poorly accepted by patients and potentially harmful. MRI needs bowel distension and intravenous contrast, it is troublesome for claustrophobic patients, and it is expensive and not available at every hospital. Numerous biomarkers have been proposed as surrogate markers of intestinal inflammation, and therefore also as potential markers of CD activity, but there is still a paucity of scientific evidence to support their use in the management and monitoring of $\mathrm{CD}$ patients.

$\mathrm{C}$-reactive protein (CRP) is an acute phase protein that has proved to be a sensitive marker of inflammation, infection, and tissue injury. CRP is produced almost exclusively in the liver on stimulation by interleukin- 6 produced at sites of inflammation. Its short half-life makes CRP a useful marker for detecting and following up CD activity. ${ }^{80,81}$ In patients with high CRP levels at diagnosis, it can be used as a very accurate marker of disease activity, and also as an independent predictor of short- and medium-term clinical relapse. ${ }^{82} \mathrm{~A}$ retrospective review of the medical charts of $104 \mathrm{CD}$ patients who had CRP assays, colonoscopy, and either small bowel follow through or CT enterography within 14 days showed that moderate-severe clinical activity (odds ratio [OR] 4.5), active disease at colonoscopy (OR 3.5), and histological evidence of severe inflammation (OR 10.6) were significantly associated with CRP elevation, whereas abnormal findings on small bowel radiographic images were not. ${ }^{83}$ Since the introduction of anti-tumor necrosis factor alpha agents, it has been well established that patients with high baseline levels of CRP have greater chances of responding to medical treatment. ${ }^{84}$ This was confirmed in a recent post hoc analysis of the ACCENT I trial: patients with high baseline CRP levels ( $\geq 0.7 \mathrm{mg} / \mathrm{dL}$ ) had a higher likelihood of remaining in remission during 1 year of infliximab maintenance therapy. ${ }^{85}$ Conversely, in a Hungarian cohort of $201 \mathrm{CD}$ patients treated with adalimumab, CRP levels at the start of the biological therapy were not associated with clinical response or remission at weeks 24 or 52, whereas early normalization of CRP (by week 12) was associated with clinical efficacy and mucosal healing. ${ }^{86}$ The "real life experience" described by Magro et al of CD patients undergoing infliximab therapy emphasized the importance of an early drop in CRP levels after starting therapy (assayed at 14 weeks) as a predictor of sustained response in the first year, irrespective of baseline serum CRP levels ${ }^{87}$ The authors also found that patients with 
more marked variations in CRP levels (CRP delta) were less likely to need further adjustments to their treatment, and that higher CRP values corresponded to a worse response. The authors judged this to be an expression of a more severe disease or a higher rate of infliximab clearance. We already know that some patients with clinically active CD (according to the CDAI) may have normal CRP levels, and that it may be difficult to know whether such patients really have active disease or only functional symptoms. Colonoscopy revealed endoscopic lesions in a sample of patients with a $\mathrm{CDAI}>150$ and $\mathrm{CRP}<5 \mathrm{mg} / \mathrm{L}$, although these lesions were only mild (CDEIS $<6$ ) in most cases. ${ }^{88}$

Fecal biomarkers, such as the neutrophil-derived calprotectin and lactoferrin, have proved useful for managing CD patients in several settings, as previously reported in two recent reviews. ${ }^{89,90}$ Both fecal calprotectin and lactoferrin significantly correlate with endoscopic and histological scores of CD activity in ileocolonic or colonic disease, but not when the disease is confined to the ileum. ${ }^{91,92}$ Calprotectin proved superior to CRP or the CDAI in discriminating between different degrees of intestinal inflammation (according to the SES-CD). ${ }^{93}$ Once disease activity has been established, fecal calprotectin can also be used to monitor the course of the disease and response to medical therapy. Mao et al conducted a meta-analysis on the ability of fecal calprotectin to predict relapse in patients with inflammatory bowel disease, finding a pooled sensitivity and specificity of $78 \%$ and $73 \%$, and comparable rates for ulcerative colitis and colonic CD. ${ }^{94}$ The use of fecal calprotectin as a surrogate marker of mucosal healing has recently been introduced in clinical trials, with a recommended cut-off of $250 \mu \mathrm{g} / \mathrm{g}$ for predicting CDEIS $\leq 3$ with $94 \%$ sensitivity and $62 \%$ specificity. ${ }^{95}$ Normalization of fecal markers was also seen to correlate with endoscopic response after therapy enhancement in 19 CD patients, while these markers remained abnormal in the majority of endoscopic nonresponders. ${ }^{96}$

In preliminary reports on the STORI trial cohort, monitoring both CRP and calprotectin (in combination) has been suggested as a way to improve accuracy in predicting mucosal healing and establishing the risk of clinical relapse within 4-6 months. ${ }^{97,98}$ Combining the two parameters also proved useful for identifying cases at higher risk of relapse within 1 year of discontinuing infliximab among patients on combined maintenance therapy with antimetabolites: $\mathrm{CRP} \geq 5.0 \mathrm{mg} / \mathrm{L}$ and fecal calprotectin $\geq 300 \mu \mathrm{g} / \mathrm{g}$ (and also male sex, no surgical resection, leukocytosis, and anemia) could identify patients at higher risk. ${ }^{99}$
In conclusion, clinicians have numerous tools for assessing disease activity in CD patients. $\mathrm{CD}$ is a complex disease, with phenotypes that may differ significantly from one patient to another, and that may even change within the same patient over time, so an algorithm appropriate for the follow-up of all CD is unlikely. A patient-tailored strategy is needed, selecting a combination of tests and procedures that best assesses disease activity (in terms of the burden of inflammation), complications, and response to therapy.

Noninvasive markers such as CRP and fecal proteins can provide baseline information to confirm any presence of intestinal inflammation, and they should be used as a preliminary step to select patients requiring further investigations. Ileocolonoscopy (with histology) remains the gold standard for assessing mucosal disease activity in the majority of patients, while a complete examination of the small intestinal mucosa using SBCE and/or balloon-assisted enteroscopy is a complementary measure to be used when proximal intestinal involvement is suspected. Imaging techniques, especially MRI, can provide information on the transmucosal activity of the disease and rule out complications. Endoscopy and imaging techniques provide complementary information and are not mutually exclusive: they should be used when there is a clinical need to reassess CD activity and extent for the purpose of adjusting or changing a patient's treatment, or for referring a patient to the surgeon, as appropriate.

\section{Disclosure}

The authors report no conflict of interest in this work.

\section{References}

1. Peyrin-Biroulet L, Loftus EV Jr, Colombel JF, Sandborn WJ. The natural history of adult Crohn's disease in population-based cohorts. Am J Gastroenterol. 2010;105(2):289-297.

2. Cosnes J, Bourrier A, Nion-Larmurier I, Sokol H, Beaugerie L, Seksik P. Factors affecting outcomes in Crohn's disease over 15 years. Gut. 2012;61(8):1140-1145.

3. Pariente B, Cosnes J, Danese S, et al. Development of the Crohn's disease digestive damage score, the Lémann score. Inflamm Bowel Dis. 2011;17(6):1415-1422.

4. Hommes D, Colombel JF, Emery P, Greco M, Sandborn WJ. Changing Crohn's disease management: need for new goals and indices to prevent disability and improve quality of life. J Crohns Colitis. 2012;6 Suppl 2: S224-S234.

5. Zallot C, Peyrin-Biroulet L. Deep remission in inflammatory bowel disease: looking beyond symptoms. Curr Gastroenterol Rep. 2013; 15(3):315.

6. Best WR, Becktel JM, Singleton JW, Kern F Jr. Development of a Crohn's disease activity index. National Cooperative Crohn's Disease Study. Gastroenterology. 1976;70(3):439-444.

7. Harvey RF, Bradshaw JM. A simple index of Crohn's-disease activity. Lancet. 1980;1(8167):514.

8. Papay P, Ignjatovic A, Karmiris K, et al. Optimising monitoring in the management of Crohn's disease: a physician's perspective. J Crohns Colitis. 2013;7(8):653-669. 
9. Tinè F, Rossi F, Sferrazza A, et al. Meta-analysis: remission and response from control arms of randomized trials of biological therapies for active luminal Crohn's disease. Aliment Pharmacol Ther. 2008;27(12):1210-1223.

10. Bouguen G, Levesque BG, Feagan BG, et al. Treat to Target: A proposed new paradigm for the management of Crohn's disease. Clin Gastroenterol Hepatol. Epub September 10, 2013.

11. Schnitzler F, Fidder H, Ferrante M, et al. Mucosal healing predicts long-term outcome of maintenance therapy with infliximab in Crohn's disease. Inflamm Bowel Dis. 2009;15(9):1295-1301.

12. Baert F, Moortgat L, Van Assche G, et al; Belgian Inflammatory Bowel Disease Research Group; North-Holland Gut Club. Mucosal healing predicts sustained clinical remission in patients with early-stage Crohn's disease. Gastroenterology. 2010;138(2):463-468.

13. Rutgeerts P, Van Assche G, Sandborn W J, et al. Adalimumab induces and maintains mucosal healing in patients with Crohn's disease: data from the EXTEND trial. Gastroenterology. 2012;142(5): 1102-1111.

14. Cellier C, Sahmoud T, Froguel E, et al. Correlations between clinical activity, endoscopic severity, and biological parameters in colonic or ileocolonic Crohn's disease. A prospective multicentre study of 121 cases. The Groupe d'Etudes Thérapeutiques des Affections Inflammatoires Digestives. Gut. 1994;35(2):231-235.

15. Allez M, Lemann M, Bonnet J, Cattan P, Jian R, Modigliani R. Long-term outcome of patients with active Crohn's disease exhibiting extensive and deep ulcerations at colonoscopy. Am J Gastroenterol. 2002;97(4):947-953.

16. Aloi M, Viola F, D'Arcangelo G, et al. Disease course and efficacy of medical therapy in stricturing paediatric Crohn's disease. Dig Liver Dis. 2013;45(6):464-468.

17. Frøslie KF, Jahnsen J, Moum BA, Vatn MH; IBSEN Group. Mucosal healing in inflammatory bowel disease: results from a Norwegian population-based cohort. Gastroenterology. 2007;133(2):412-422.

18. Af Bjorkesten CG, Nieminen U, Sipponen T, Turunen U, Arkkila P, Farkkila M. Mucosal healing at 3 months predicts long-term endoscopic remission in anti-TNF-treated luminal Crohn's disease. Scand J Gastroenterol. 2013;48(5):543-551.

19. Hanauer SB, Feagan BG, Lichtenstein GR, et al; ACCENT I Study Group. Maintenance infliximab for Crohn's disease: the ACCENT I randomised trial. Lancet. 2002;359(9317):1541-1549.

20. Benitez JM, Meuwis MA, Reenaers C, Van Kemseke C, Meunier P, Louis E. Role of endoscopy, cross-sectional imaging and biomarkers in Crohn's disease monitoring. Gut. 2013;62(12):1806-1816.

21. De Cruz P, Kamm MA, Prideaux L, Allen PB, Moore G. Mucosal healing in Crohn's disease: a systematic review. Inflamm Bowel Dis. 2013;19(2):429-444.

22. Mazzuoli S, Guglielmi FW, Antonelli E, Salemme M, Bassotti G, Villanacci V. Definition and evaluation of mucosal healing in clinical practice. Dig Liver Dis. 2013;45(12):969-977.

23. Mary JY, Modigliani R. Development and validation of an endoscopic index of the severity for Crohn's disease: a prospective multicentre study. Groupe d'Etudes Therapeutiques des Affections Inflammatoires du Tube Digestif (GETAID). Gut. 1989;30(7):983-989.

24. Daperno M, D’Haens G, Van Assche G, et al. Development and validation of a new, simplified endoscopic activity score for Crohn's disease: the SES-CD. Gastrointest Endosc. 2004;60(4):505-512.

25. Rutgeerts P, Geboes K, Vantrappen G, Beyls J, Kerremans R, Hiele M. Predictability of the postoperative course of Crohn's disease. Gastroenterology. 1990;99(4):956-963.

26. Sipponen T, Nuutinen H, Turunen U, Färkkilä M. Endoscopic evaluation of Crohn's disease activity: comparison of the CDEIS and the SES-CD. Inflamm Bowel Dis. 2010;16(12):2131-2136.

27. Ferrante M, Colombel JF, Sandborn WJ, et al; International Organization for the Study of Inflammatory Bowel Diseases. Validation of endoscopic activity scores in patients with Crohn's disease based on a post hoc analysis of data from SONIC. Gastroenterology. 2013;145(5): 978-986.
28. Rutgeerts P, Geboes K, Vantrappen G, Kerremans R, Coenegrachts JL, Coremans G. Natural history of recurrent Crohn's disease at the ileocolonic anastomosis after curative surgery. Gut. 1984;25(6):665-672.

29. Rutgeerts P. Review article: recurrence of Crohn's disease after surgery - the need for treatment of new lesions. Aliment Pharmacol Ther. 2006;24 Suppl 3:29-32.

30. Villanacci V, Antonelli E, Geboes K, Casella G, Bassotti G. Histological healing in inflammatory bowel disease: a still unfulfilled promise. World J Gastroenterol. 2013;19(7):968-978.

31. D'Haens G, Van Deventer S, Van Hogezand R, et al. Endoscopic and histological healing with infliximab anti-tumor necrosis factor antibodies in Crohn's disease: a European multicenter trial. Gastroenterology. 1999;116(5):1029-1034.

32. Smith JP, Bingaman SI, Ruggiero F, et al. Therapy with the opioid antagonist naltrexone promotes mucosal healing in active Crohn's disease: a randomized placebo-controlled trial. Dig Dis Sci. 2011;56(7): 2088-2097.

33. Bourreille A, Ignjatovic A, Aabakken L, et al; World Organization of Digestive Endoscopy (OMED) and the European Crohn's and Colitis Organisation (ECCO). Role of small-bowel endoscopy in the management of patients with inflammatory bowel disease: an international OMED-ECCO consensus. Endoscopy. 2009;41(7):618-637.

34. Eliakim R, Fischer D, Suissa A, et al. Wireless capsule video endoscopy is a superior diagnostic tool in comparison to barium follow-through and computerized tomography in patients with suspected Crohn's disease. Eur J Gastroenterol Hepatol. 2003;15(4):363-367.

35. Albert JG, Martiny F, Krummenerl A, et al. Diagnosis of small bowel Crohn's disease: a prospective comparison of capsule endoscopy with magnetic resonance imaging and fluoroscopic enteroclysis. Gut. 2005;54(12):1721-1727.

36. Pons Beltrán V, Nos P, Bastida G, et al. Evaluation of postsurgical recurrence in Crohn's disease: a new indication for capsule endoscopy? Gastrointest Endosc. 2007;66(3):533-540.

37. Efthymiou A, Viazis N, Mantzaris G, et al. Does clinical response correlate with mucosal healing in patients with Crohn's disease of the small bowel? A prospective, case-series study using wireless capsule endoscopy. Inflamm Bowel Dis. 2008;14(11):1542-1547.

38. Yang L, Ge ZZ, Gao YJ, et al. Assessment of capsule endoscopy scoring index, clinical disease activity, and C-reactive protein in small bowel Crohn's disease. J Gastroenterol Hepatol. 2013;28(5):829-833.

39. Niv Y, Ilani S, Levi Z, et al. Validation of the Capsule Endoscopy Crohn's Disease Activity Index (CECDAI or Niv score): a multicenter prospective study. Endoscopy. 2012;44(1):21-26.

40. Gralnek IM, Defranchis R, Seidman E, Leighton JA, Legnani P, Lewis BS. Development of a capsule endoscopy scoring index for small bowel mucosal inflammatory change. Aliment Pharmacol Ther. 2008;27(2):146-154.

41. Koulaouzidis A, Douglas S, Plevris JN. Lewis score correlates more closely with fecal calprotectin than Capsule Endoscopy Crohn's Disease Activity Index. Dig Dis Sci. 2012;57(4):987-993.

42. Panes J, Bouhnik Y, Reinisch W, et al. Imaging techniques for assessment of inflammatory bowel disease: joint ECCO and ESGAR evidencebased consensus guidelines. J Crohns Colitis. 2013;7(7):556-585.

43. Panes J, Bouzas R, Chaparro M, et al. Systematic review: the use of ultrasonography, computed tomography and magnetic resonance imaging for the diagnosis, assessment of activity and abdominal complications of Crohn's disease. Aliment Pharmacol Ther. 2011;34(2):125-145.

44. Maconi G, Radice E, Greco S, Bianchi Porro G. Bowel ultrasound in Crohn's disease. Best Pract Res Clin Gastroenterol. 2006;20(1): 93-112.

45. Di Mizio R, Maconi G, Romano S, D’Amario F, Bianchi Porro G, Grassi R. Small bowel Crohn disease: sonographic features. Abdom Imaging. 2004;29(1):23-35.

46. Parente F, Greco S, Molteni M, et al. Oral contrast enhanced bowel ultrasonography in the assessment of small intestine Crohn's disease. A prospective comparison with conventional ultrasound, $\mathrm{x}$-ray studies, and ileocolonoscopy. Gut. 2004;53(11):1652-1657. 
47. Bru C, Sans M, Defelitto MM, et al. Hydrocolonic sonography for evaluating inflammatory bowel disease. AJR Am J Roentgenol. 2001;177(1):99-105.

48. Migaleddu V, Scanu AM, Quaia E, et al. Contrast-enhanced ultrasonographic evaluation of inflammatory activity in Crohn's disease. Gastroenterology. 2009;137(1):43-52.

49. Ripolles T, Martinez MJ, Paredes JM, Blanc E, Flors L, Delgado F. Crohn disease: correlation of findings at contrast enhanced US with severity at endoscopy. Radiology. 2009;253(1):241-248.

50. De Franco A, Marzo M, Felice C, et al. Ileal Crohn's disease: CEUS determination of activity. Abdom Imaging. 2012;37(3):359-368.

51. Calabrese E, Zorzi F, Zuzzi S, et al. Development of a numerical index quantitating small bowel damage as detected by ultrasonography in Crohn's disease. J Crohns Colitis. 2012;6(8):852-860.

52. Park MJ, Lim JS. Computed tomography enterography for evaluation of inflammatory bowel disease. Clin Endosc. 2013;46(4):327-366.

53. Fiorino G, Bonifacio C, Peyrin-Biroulet L, et al. Prospective comparison of computed tomography enterography and magnetic resonance enterography for assessment of disease activity and complications in ileocolonic Crohn's disease. Inflamm Bowel Dis. 2011;17(5): 1073-1080

54. Wu YW, Tang YH, Hao NX, Tang CY, Miao F. Crohn's disease: CT enterography manifestations before and after treatment. Eur J Radiol. 2012;81(1):52-59.

55. Siddiki HA, Fidler JL, Fletcher JG, et al. Prospective comparison of state-of-the-art MR enterography and CT enterography in small-bowel Crohn's disease. AJR Am J Roentgenol. 2009;193(1):113-121.

56. Lee SS, Kim AY, Yang SK, et al. Crohn disease of the small bowel: comparison of CT enterography, MR enterography, and small-bowel follow-through as diagnostic techniques. Radiology. 2009;251(3): $751-761$.

57. Masselli G, Gualdi G. CT and MR enterography in evaluating small bowel diseases: when to use which modality? Abdom Imaging. 2013;38(2):249-259.

58. Craig O, O'Neill S, O’Neill F, et al. Diagnostic accuracy of computed tomography using lower doses of radiation for patients with Crohn's disease. Clin Gastroenterol Hepatol. 2012;10(8):886-892.

59. Lee SJ, Park SH, Kim AY, et al. A prospective comparison of standard-dose CT enterography and 50\% reduced-dose CT enterography with and without noise reduction for evaluating Crohn disease. AJR Am J Roentgenol. 2011;197(1):50-57.

60. Cipriano LE, Levesque BG, Zaric GS, Loftus EV Jr, Sandborn WJ. Costeffectiveness of imaging strategies to reduce radiation-induced cancer risk in Crohn's disease. Inflamm Bowel Dis. 2012;18(7):1240-1248.

61. Feuerbach S. MRI enterography: the future of small bowel diagnostics? Dig Dis. 2010;28(3):433-438.

62. Kuehle CA, Ajaj W, Ladd SC, Massing S, Barkhausen J, Lauenstein TC. Hydro-MRI of the small bowel: effect of contrast volume, timing of contrast administration, and data acquisition on bowel distention. AJR Am J Roentgenol. 2006;187:W375-W385.

63. Negaard A, Paulsen V, Sandvik L, et al. A prospective randomized comparison between two MRI studies of the small bowel in Crohn's disease, the oral contrast method and MR enteroclysis. Eur Radiol. 2007;17(9):2294-2301

64. Masselli G, Gualdi G. MR imaging of the small bowel. Radiology. 2012;264(2):333-348.

65. Makanyanga JC, Taylor SA. Current and future role of MR enterography in the management of Crohn disease. AJR Am J Roentgenol. 2013;201(1):56-64.

66. Al-Hawary, Zimmermann EM. A new look at Crohn's disease: novel imaging techniques. Curr Opin Gastroenterol. 2012;28(4):334-340.

67. Rimola J, Ordás I, Rodríguez S, Ricart E, Panés J. Imaging indexes of activity and severity for Crohn's disease: current status and future trends. Abdom Imaging. 2012;37(6):958-966.

68. Rimola J, Rodríguez S, García-Bosch O, et al. Magnetic resonance for assessment of disease activity and severity in ileocolonic Crohn's disease. Gut. 2009;58(8):1113-1120.
69. Rimola J, Ordás I, Rodríguez S, et al. Magnetic resonance imaging for evaluation of Crohn's disease: validation of parameters of severity and quantitative index of activity. Inflamm Bowel Dis. 2011;17(8):1759-1768.

70. Steward MJ, Punwani S, Proctor I, et al. Nonperforating small bowel Crohn's disease assessed by MRI enterography: derivation and histopathological validation of an MR-based activity index. Eur $J$ Radiol. 2012;81(9):2080-2088.

71. Tielbeek JA, Makanyanga JC, Bipat S, et al. Grading Crohn Disease activity with MRI: interobserver variability of MRI features, MRI scoring of severity, and correlation with Crohn Disease Endoscopic Index of Severity. AJR Am J Roentgenol. 2013;201(6):1220-1228.

72. Sempere GA, Martinez Sanjuan V, Medina Chulia E, et al. MRI evaluation of inflammatory activity in Crohn's disease. AJR Am J Roentgenol. 2005;184(6):1829-1835.

73. Ordàs I, Rimola J, Ripolles T, et al. Accuracy of MRI to assess therapeutic responses and mucosal healing in Crohn's disease. Gastroenterology. 2011;140(Suppl 1):S73.

74. Van Assche G, Herrmann KA, Louis E, et al. Effects of infliximab therapy on transmural lesions as assessed by magnetic resonance enteroclysis in patients with ileal Crohn's disease. J Crohns Colitis. 2013;7(12):950-957.

75. Lawrance IC, Welman CJ, Shipman P, Murray K. Correlation of MRI-determined small bowel Crohn's disease categories with medical response and surgical pathology. World J Gastroenterol. 2009;15(27): $3367-3375$

76. Zappa M, Stefanescu C, Cazals-Hatem D, et al. Which magnetic resonance imaging findings accurately evaluate inflammation in small bowel Crohn's disease? A retrospective comparison with surgical pathologic analysis. Inflamm Bowel Dis. 2011;17(4):984-993.

77. Pariente B, Peyrin-Biroulet L, Cohen L, et al. Gastroenterology review and perspective: the role of cross-sectional imaging in evaluating bowel damage in Crohn disease. AMJ Am J Roentegenol. 2011;197(1): $42-49$.

78. Van Assche G, Vanbeckevoort D, Bielen D, et al. Magnetic resonance imaging of the effects of infliximab on perianal fistulizing Crohn's disease. Am J Gastroenterol. 2003;98(2):332-339.

79. Karmiris K, Bielen D, Vanbeckevoort D, et al. Long-term monitoring of infliximab therapy for perianal fistulizing Crohn's disease by using magnetic resonance imaging. Clin Gastroenterol Hepatol. 2011;9(2):130-136.

80. Vermeire S, Van Assche G, Rutgeerts P. C-reactive protein as a marker for inflammatory bowel disease. Inflamm Bowel Dis. 2004;10(5): 661-665.

81. Chamouard P, Richert Z, Meyer N, Rahmi G, Baumann R. Diagnostic value of C-reactive protein for predicting activity level of Crohn's disease. Clin Gastroenterol Hepatol. 2006;4(7):882-887.

82. Kiss LS, Papp M, Lovasz BD, et al. High-sensitivity C-reactive protein for identification of disease phenotype, active disease, and clinical relapses in Crohn's disease: a marker for patient classification? Inflamm Bowel Dis. 2012;18(9):1647-1654.

83. Solem CA, Loftus EV Jr, Tremaine WJ, Harmsen WS, Zinsmeister AR, Sandborn WJ. Correlation of C-reactive protein with clinical, endoscopic, histologic, and radiographic activity in inflammatory bowel disease. Inflamm Bowel Dis. 2005;11(8):707-712.

84. Louis $\mathrm{E}$, Vermeire $\mathrm{S}$, Rutgeerts $\mathrm{P}$, et al. A positive response to infliximab in Crohn disease: association with a higher systemic inflammation before treatment but not with-308 TNF gene polymorphism. Scand $J$ Gastroenterol. 2002;37(7):818-824.

85. Reinisch W, Wang Y, Oddens BJ, Link R. C-reactive protein, an indicator for maintained response or remission to infliximab in patients with Crohn's disease: a post-hoc analysis from ACCENT I. Aliment Pharmacol Ther. 2012;35(5):568-576.

86. Kiss LS, Szamosi T, Molnar T, et al; Hungarian IBD Study Group. Early clinical remission and normalisation of CRP are the strongest predictors of efficacy, mucosal healing and dose escalation during the first year of adalimumab therapy in Crohn's disease. Aliment Pharmacol Ther. 2011;34(8):911-922. 
87. Magro F, Rodrigues-Pinto E, Santos-Antunes J, et al. High C-reactive protein in Crohn's disease patients predicts nonresponse to infliximab treatment. J Crohns Colitis. 2014;8(2):129-136.

88. Denis M A, Reenaers C, Fontaine F, Belaiche J, Louis E. Assessment of endoscopic activity index and biological inflammatory markers in clinically active Crohn's disease with normal C-reactive protein serum level. Inflamm Bowel Dis. 2007;13(9):1100-1105.

89. Caccaro R, D’Incá R, Sturniolo GC. Clinical utility of calprotectin and lactoferrin as markers of inflammation in patients with inflammatory bowel disease. Expert Rev Clin Immunol. 2010;6(4):551-558.

90. Caccaro R, D'Incà R, Pathak S, Sturniolo GC. Clinical utility of calprotectin and lactoferrin in patients with inflammatory bowel disease: is there something new from the literature? Expert Rev Clin Immunol. 2012;8(6):579-585.

91. Sipponen T, Karkkainen P, Savilahti E, et al. Correlation of faecal calprotectin and lactoferrin with an endoscopic score for Crohn's disease and histological findings. Aliment Pharmacol Ther. 2008;28(10): 1221-1229.

92. Sipponen T, Savilahti E, Kohlo KL, Nuutinen H, Turunen U, Farkkila M. Crohn's disease activity assessed by fecal calprotectin and lactoferrin: correlation with Crohn's disease activity index and endoscopic findings. Inflamm Bowel Dis. 2008;14(1):40-46.

93. Schoepfer AM, Beglinger C, Straumann A, et al. Fecal calprotectin correlates more closely with the simple endoscopic score for Crohn's disease (SES-CD) than CRP, blood leukocytes, and the CDAI. Am J Gastroenterol. 2010;105(1):162-169.
94. Mao R, Xiao YL, Gao X, et al. Fecal calprotectin in predicting relapse of inflammatory bowel diseases: a meta-analysis of prospective studies. Inflamm Bowel Dis. 2012;18(10):1894-1899.

95. D'Haens G, Ferrante M, Vermeire S, et al. Fecal calprotectin is a surrogate marker for endoscopic lesions in inflammatory bowel disease. Inflamm Bowel Dis. 2012;18(12):2218-2224.

96. Sipponen T, Björkesten CG, Färkkilä M, Nuutinen H, SavilahtiE, Kolho KL. Faecal calprotectin and lactoferrin are reliable surrogate markers of endoscopic response during Crohn's disease treatment. Scand J Gastroenterol. 2010;45(3):325-331.

97. Lemann M, Colombel JF, Grimaud JC, et al. Fecal calprotectin and high sensitivity $\mathrm{C}$-reactive protein levels to predict mucosal healing in patients with Crohn's disease. A subanalysis of the STORI study. Gut. 2010;59(Suppl III):A80.

98. de Suray N, Salleron J, Vernier-Massouille G, et al. Close monitoring of CRP and fecal calprotectin levels to predict relapse in Crohn's disease patients. A sub-analysis of the STORI study. J Crohn Colitis. 2012;6(1):P274.

99. Louis E, Mary JY, Vernier-Massouille G, et al; Groupe D'etudes Therapeutiques Des Affections Inflammatoires Digestives. Maintenance of remission among patients with Crohn's disease on antimetabolite therapy after infliximab therapy is stopped. Gastroenterology. 2012;142(1):63-70.
Clinical and Experimental Gastroenterology

\section{Publish your work in this journal}

Clinical and Experimental Gastroenterology is an international, peerreviewed, open access journal, publishing all aspects of gastroenterology in the clinic and laboratory, including: Pathology, pathophysiology of gastrointestinal disease; Investigation and treatment of gastointestinal disease; Pharmacology of drugs used in the alimentary tract;

\section{Dovepress}

Immunology/genetics/genomics related to gastrointestinal disease. This journal is indexed on CAS. The manuscript management system is completely online and includes a very quick and fair peer-review system. Visit http://www.dovepress.com/testimonials.php to read real quotes from published authors.

Submit your manuscript here: http://www.dovepress.com/clinical-and-experimental-gastroenterology-journal 